\title{
A Current Commentary on Breech Vaginal Deliveries: The Changing Landscape of Patient versus Physician Autonomy
}

\author{
Meghan G. Hill, MBBS ${ }^{1}$ \\ ${ }^{1}$ Division of Maternal-Fetal Medicine, Department of Obstetrics and \\ Gynecology, The University of Arizona, Tucson, Arizona \\ Am J Perinatol Rep 2019;9:e185-e189.
}

\section{Clinical Case}

A 38-year old G2P1001 presented for a second opinion at 34 weeks of gestational age. Her obstetric history included a term vaginal delivery of a 3,460 g infant complicated by a postpartum hemorrhage requiring the administration of oxytocin, methergine, hemabate, misoprostol, and curettage for resolution. Her current pregnancy was a spontaneous dichorionic diamniotic twin pregnancy. The fetuses were concordantly grown and the pregnancy had otherwise been uncomplicated. She sought a new obstetrician as her physician had recommended a cesarean for breech/breech presentation and declined to offer a vaginal delivery in this circumstance.

The risks and benefits of a primary cesarean delivery, an attempted version of the first twin, and an attempt at breech/ breech vaginal delivery were discussed. Potential complications were discussed with the patient to include cord prolapse, fetal distress, head entrapment, fetal death, and neonatal developmental delay. The potential need for Dührssen's incisions, forcep delivery of the aftercoming fetal head and the unlikely event of twin locking should the second fetus become cephalic were discussed. The American College of Obstetricians and Gynecologists (ACOG) guidelines recommending cesarean in her situation were reviewed, as was the paucity of trial data regarding breech/breech twin deliveries. The provider's experience with breech vaginal delivery was discussed with the patient. She was given an approximate 30\% risk of requiring emergency cesarean delivery. The patient was advised that the provider thought her chance of requiring forceps for the delivery of one or both twins was likely also approximately 30\% and informed that these estimates were based on the provider's experience with the proposed procedure. The patient decided to pursue vaginal delivery.

The patient developed severe cholestasis of pregnancy with her total bile acids returning at a value of $96 \mathrm{umol} / \mathrm{L}$ at $36^{5 / 7}$

received

December 29, 2018 accepted after revision February 8, 2019
Address for correspondence Meghan G. Hill, MBBS, Division of Maternal-Fetal Medicine, Department of Obstetrics and Gynecology, The University of Arizona, 1501 North Campbell Avenue, Tucson, AZ, 85724 (e-mail: meghanhill@obgyn.arizona.edu).

weeks of gestation. Delivery was recommended. The fetuses were in complete breech (Twin A) and transverse (Twin B) presentations. She chose an induction of labor. Her starting Bishop score was 8. An oxytocin infusion was commenced. During a cervical examination at $6 \mathrm{~cm}$ dilatation, 70\% effacement, and -1 station, the patient's membranes ruptured with confirmation of fetus $A$ in a complete breech presentation with the right fetal foot, the fetal genitalia and the sacrum presenting. No umbilical cord was palpable. Epidural anesthesia was administered. The patient progressed over the next several hours to complete dilatation and was allowed to "labor down" for approximately 45 minutes at which time she was transferred to the operating room for delivery. She delivered Twin A, a complete breech fetus, without the need for assistance with forceps. Newborn was a 3,090 g male with Apgar scores of 7 and 8 . A breech extraction, in combination with maternal expulsive efforts, was performed of twin B which presented as a frank breech. Again forceps were not required. The newborn was a 2,750 g female with Apgar scores of 9 and 9. In spite of active management of the third stage, the uterus did not readily contract. At placental separation the patient began bleeding and uterine atony was diagnosed. At 5 minutes after placental delivery, the provider estimated blood loss to be at least $1,000 \mathrm{~mL}$. The atony was refractory to oxytocin bolus, hemabate, methergine, misoprostol, tranexamic acid, and continued bimanual massage. The anesthesiologist called to have blood products available and a second obstetrician was made aware that a postpartum hemorrhage had been diagnosed. A Bakri balloon was placed and inflated to $500 \mathrm{~mL}$. The estimated blood loss was $1,300 \mathrm{~mL}$ at this time and bleeding resolved. Laboratory testing was performed and a stable maternal fibrinogen was noted at $861 \mathrm{mg} / \mathrm{dL}$. The total hemoglobin drop over serial laboratory examinations over 6 hours was 5 (from 15.4 to $10 \mathrm{~g} / \mathrm{dL}$ ). The Bakri was removed the following morning without recurrence of bleeding. Both

Copyright @ 2019 by Thieme Medical Publishers, Inc., 333 Seventh Avenue, New York, NY 10001, USA. Tel: +1(212) 584-4662.
License terms

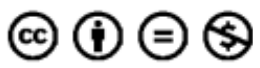

DOI https://doi.org/ 10.1055/s-0039-1692178. ISSN 2157-6998. 
babies and mother were clinically stable and were discharged from the hospital on day 2 after delivery.

This case highlights a quandary: a patient makes a request for vaginal delivery and seeks a provider willing to support her in her decision. The immediate question is whether this is a reasonable request (is practice outside of guidelines in this case acceptable)? Secondarily, which party's autonomy should be paramount in this situation, the physician's or the patient's?

\section{Discussion}

"Planned Caesarean Section versus Planned Vaginal Birth for Breech Presentation at term: A Randomized Multicenter Trial," published in the year $2000^{1}$ has had profound and at times unintended consequences on women and their infants. The investigators randomized 2088 women in 26 countries with singleton fetuses in frank or complete breech presentations to a planned cesarean or planned attempt at vaginal birth. Most women in the trial remained in the group to which they were assigned and outcomes were assessed on 2078 of the participants. The trial was a major achievement and one of its size will likely never be repeated in relation to the question of the safety of breech vaginal birth. Women randomized to planned vaginal delivery had a $36.1 \%$ chance of cesarean delivery if they attempted a vaginal delivery, were more likely to experience a cord prolapse, fetal heart rate abnormalities, difficult delivery of the fetal head, and most importantly, a stillbirth or a neonatal death. Maternal outcomes were equivalent whether they were delivered via cesarean or vaginally. An obvious difference is that all women delivering via cesarean were exposed to a surgical procedure, with its attendant risks, whereas those who were allocated to vaginal delivery only had a $36.1 \%$ chance of this outcome.

In spite of the high integrity of the study, there were limitations. The providers participating in the study had to self-identify as experienced in breech vaginal delivery with the agreement of the head of their department. This study led to many institutions in developed countries increasing the number of breech deliveries they did as they were participating in the protocol which has been a criticism of the work (the suspicion being that perhaps providers who would have otherwise recommended a cesarean were allowing an attempt at vaginal delivery if a patient was randomized into that group). ${ }^{2}$ The investigators noted that the neonatal benefits of cesarean delivery were greater in high resource countries. $^{1}$ There are multiple potential reasons for this; however, a consideration would be that providers in lowresource settings may in fact have more experience with breech vaginal delivery. ${ }^{2}$ Conversely, cesarean may be less safe in low-resource settings.

The conclusion reached by many after review of this rigorously-implemented trial was that cesarean delivery should be recommended in cases of breech presentation. Indeed ACOG recommended that women undergo cesarean delivery in the setting of a term breech fetus in 2001. Many other high and middle income countries followed, with the rates of breech vaginal delivery falling from 80 to $50 \%$ in the
Netherlands shortly after the trial was published. ${ }^{3}$ It is curious that one trial changed practice so dramatically.

In light of follow-up publications by trial authors and multiple others regarding the outcomes of women and neonates after breech vaginal birth and the limitations of the study, ${ }^{2,4-7}$ ACOG made new recommendations in 2006 regarding the acceptability of breech vaginal delivery. The specific findings that seemed to sway opinion were that when many of the children delivered in the initial trial by Hannah et al were reassessed at 2 years of age, there were not differences in outcomes irrespective of mode of delivery. ${ }^{5}$ Additionally, several authors published series of patients that had delivered vaginally without the same apparent risk of mortality for the fetus as seen in the Hannah trial., ${ }^{6,7}$

The 2006 ACOG committee opinion, recently updated in $2018,{ }^{8}$ recommends several criteria which have not been rigorously tested in trials. The author suggests that several of them never should be, rather that there is likely an elevated risk with breech vaginal delivery, even in experienced hands. Providers should explain this and divulge their experience and confidence with breech vaginal deliveries to patients and allow them to exercise their autonomy regarding their preferred mode of delivery. The ACOG recommendations are discussed below:

(1) "The decision regarding the mode of delivery should consider patient wishes and the experience of the health care provider."

The safety of breech vaginal delivery in relation to provider experience has never been (and likely will never be) prospectively studied as the primary outcome in any study. The level of experience may mean different things. For example, a provider may have delivered 10 breech fetuses, none with the need for forceps. This provider may be less equipped to deal with fetal head entrapment than a provider who has delivered only five breech fetuses, three of them with Piper forceps. The individual dexterity of the provider cannot be controlled for. For some providers five breech deliveries may be enough, whereas for others, 20 is insufficient. In our case, the provider supervised a resident during these deliveries, continuing the education in the method of breech vaginal delivery. This opportunity to deliver two breech fetuses consecutively is a rare one and likely provides valuable tactile learning for when an unexpected breech presents later in that resident's career. That is to say, the experience of delivering a vaginal breech is valuable, even in trainees who will go into practice not planning to deliver breeches vaginally, as will the majority of graduates currently in training. The author notes that difficult delivery of the fetal head and other problems, such as a nuchal arm can occur at cesarean also, ${ }^{1}$ so many of the maneuvers required for safe delivery of a breech apply to both types of delivery.

(2) "Obstetrician-gynecologists and other obstetric care providers should offer external cephalic version as an alternative to planned cesarean for a woman who has a term singleton breech fetus, desires a planned 
vaginal delivery of a vertex-presenting fetus, and has no contraindications. External cephalic version should be attempted only in settings in which cesarean delivery services are readily available."

The author agrees with this statement and indeed performs the procedure. It is one of the three options offered to patients ${ }^{9}$ and some patients elect to undergo an attempt at a vaginal delivery without an external cephalic version which the author believes is an acceptable choice. ACOG also recommends referral to other providers in the case where patients wish to undergo a procedure the clinician is uncomfortable to provide (primary elective cesarean delivery without a medical indication, or referring for abortion care, for example). ${ }^{10}$ If a provider is uncomfortable performing an external cephalic version or a breech vaginal delivery and the patient desires one of these procedures, referral so that the patient may access the desired procedure should be considered. ${ }^{10}$

(3) "Planned vaginal delivery of a term singleton breech fetus may be reasonable under hospital-specific protocol guidelines for eligibility and labor management."

This recommendation seems based on large patient cohorts published after the trial by Hannah., ${ }^{6,7}$ Several studies since have assisted in predicting which deliveries are more likely to entail neonatal complications. ${ }^{11}$ While the author agrees that clinical judgement should be used, the underlying assumption is that if the patient falls outside of the protocol, they have no option other than a cesarean delivery. Most patients are willing to undergo a cesarean if it is recommended. However, we should include in our considerations of each individual patient that no protocol is specifically engineered for them. The patient in the case discussed here, for example, likely would not have met criteria for a hospital protocol and would effectively have been compelled to have a cesarean. Additionally, though good results have been noted in cohorts when induction of labor is not permitted and labor must progress in a timely fashion, this does not mean that less favorable results would occur with induced labor or more patience with the labor in general. Multiple studies have now indicated that women with cephalic-presenting fetuses should be given more time to labor. ${ }^{12,13}$ The same may be true for breech fetuses. Indeed, permitting more liberal induction of labor may actually decrease fetal and neonatal morbidity and mortality as it may result in breech fetuses delivering when the most skilled attendants are available, rather than when whoever is on call is available. It also may make providers with experience more willing to perform the deliveries rather than being constantly available for weeks as they await the patient's spontaneous labor. Lastly, the reality is that many hospitals have financial interests that would introduce bias into decisions regarding higher-risk deliveries and the creation of protocols. It may be that insurers and hospitals would prefer to take on the legal and financial risks of a planned cesarean over a breech vaginal delivery. A recommendation regarding using a hospital protocol may be more about the comfort of risk managers and insurers than the promotion of the health of women and their babies. A more valid question may be whether or not insurers should be able to influence decisions made by a patient and physician.

(4) "If a vaginal breech delivery is planned, a detailed informed consent should be documented, including risks that perinatal or neonatal mortality or shortterm serious neonatal morbidity may be higher than if a cesarean delivery is planned."

The author agrees that informed consent should be sought for every procedure, including breech vaginal delivery.

An additional recommendation regarding breech delivery of second twins (discussing the safety and appropriateness of this approach as desired) was included in the 2006 ACOG committee opinion which has now been removed. ${ }^{8}$ As far as the author is aware, there are no trials specifically assessing mode of delivery for breech/breech presentations in twins and suspects that one will not be performed. However, the author does have experience with breech vaginal delivery and with breech-presenting first twins. Aside from the unlikely possibility of twin locking that would require version of the second twin to a cephalic presentation, it seems that the risks of breech/breech vaginal delivery would apply mostly to the first, or presenting twin, making it no less safe that singleton breech vaginal delivery. The author notes that ACOG guidelines recommend an attempt at vaginal delivery only if the presenting twin is vertex. ${ }^{14}$

The decision to act in the best interest of a patient may be outside of ACOG guidelines, ${ }^{15}$ as this may be preferable to the alternative, which is in many states is a high-risk home birth. This patient accessed the opportunity for a vaginal delivery only by seeking out a physician who would provide it through word-of-mouth (the author believes that this patient would have ultimately unhappily accepted a cesarean if forced to choose between surgery and a home birth, but not all patients will). Conversely, physicians frequently are put in a position of considering their own autonomy in these situations. Are they willing to take the risk of the personal and professional consequences of a delivery that does not go well when they are practicing differently, even with good documentation? Many physicians understandably find the risks to themselves to be too high and exercise their own professional autonomy, declining to respect the autonomy of the patient.

If trained providers in medical facilities are unwilling to provide care that is acceptable to their patients, alternative routes will be sought. This has in fact been seen in this provider's state. In Arizona, home birth is permissible by licensed midwives. Legally, a midwife may also perform breech vaginal deliveries in a home setting, along with vaginal birth after cesarean delivery, though delivery of multiples is considered a contraindication to home delivery. ${ }^{16}$ Lawmakers are not subject to following ACOG guidelines and hence, legislated to allow home birth in situations specifically cited to be contraindications to home birth by ACOG. ${ }^{17}$ This is not to say that all providers that offer home birth will agree to perform these deliveries (the author has had several midwives bring patients laboring breeches to the hospital for a physician- 
supervised delivery). However, permissive laws in combination with restrictive guidelines can have devastating effects for women and their families. Home birth is known to increase the risk of neonatal morbidity and mortality. ${ }^{17,18}$ Importantly in the instance of maternal postpartum hemorrhage access to emergency surgery and blood products are vital to prevent maternal mortality, and neither of these options are available with home birth. Tragically, mothers can die from hemorrhage in home birth settings awaiting the arrival of emergency medical care. ${ }^{19}$ And while there are benefits of the interventions in our hospitals, cesarean deliveries (what was recommended to this patient) more than triple the risk of maternal mortality and have long-term consequences, especially in repeat pregnancies. ${ }^{20-22}$ It is acceptable for a patient to weigh risks with all information available and request a vaginal delivery in the safest setting possible. It is ethically required that we as providers allow autonomy and minimize risk. In the case presented here, the patient had a life-threatening hemorrhage and the author is skeptical that she would have arrived at the hospital in time to avoid severe maternal morbidity or mortality.

Lastly, the management of this patient should be open to discussion. Procedures are learned during an apprenticeship of sorts (for the author, a residency and fellowship during which they had multiple mentors who taught twin, forcep and breech vaginal deliveries). Several parts of the patient management were based on combinations of styles of the author's predecessors. Letting a breech "labor down" to decrease the risks of a residual cervical lip, the correct approach to placement of forceps, the desire to deliver all breeches and twins in the operating room were all elements of training that the author accepted as safe practice. However, that is not to say that this is the only or safest approach. Many of these elements will likely never be prospectively studied. As we face a climate of increasing litigation, our approach to teaching our apprentices may need to change, and this article does not mean to provide a solution to the question of how effective continuing education of breech vaginal deliveries should be performed.

No provider should be put in the situation of performing a procedure that they do not feel is safe or that they worry will create an unacceptable risk of litigation. However, the cesarean delivery rate we see is likely at least in part related to selfprotection on the part of physicians. We do need to respect that patients have the right to make their own decisions, even if we perceive them to be a bad decisions. ${ }^{23}$ We are much more comfortable with this, however, when active management is not involved (refusal of a medically-indicated induction, for example, does not actually require the physician to perform a procedure). In this case, the provider thought that the patient was making a considered decision and agreed that the attempt at a vaginal delivery was reasonable. It is fortunate that she delivered in the operating room of a hospital with availability of appropriate treatment for her postpartum hemorrhage. Providers and hospitals should consider that in protecting our own interests, refusal to provide coverage for higher risk procedures can place patients at risk.

\section{Disclosure}

The author has no financial support and no conflicts of interest to disclose.

\section{Conflicts of Interest}

None.

\section{References}

1 Hannah ME, Hannah WJ, Hewson SA, Hodnett ED, Saigal S, Willan AR; Term Breech Trial Collaborative Group. Planned caesarean section versus planned vaginal birth for breech presentation at term: a randomised multicentre trial. Lancet 2000;356(9239):1375-1383

2 Kotaska A. Inappropriate use of randomised trials to evaluate complex phenomena: case study of vaginal breech delivery. BMJ 2004;329(7473):1039-1042

3 Rietberg CC, Elferink-Stinkens PM, Visser GH. The effect of the Term Breech Trial on medical intervention behaviour and neonatal outcome in The Netherlands: an analysis of 35,453 term breech infants. BJOG 2005;112(02):205-209

4 Hannah ME, Whyte H, Hannah WJ, et al; Term Breech Trial Collaborative Group. Maternal outcomes at 2 years after planned cesarean section versus planned vaginal birth for breech presentation at term: the international randomized Term Breech Trial. Am J Obstet Gynecol 2004;191(03):917-927

5 Whyte H, Hannah ME, Saigal S, et al; Term Breech Trial Collaborative Group. Outcomes of children at 2 years after planned cesarean birth versus planned vaginal birth for breech presentation at term: the International Randomized Term Breech Trial. Am J Obstet Gynecol 2004;191(03):864-871

6 Giuliani A, Schöll WM, Basver A, Tamussino KF. Mode of delivery and outcome of 699 term singleton breech deliveries at a single center. Am J Obstet Gynecol 2002;187(06):1694-1698

7 Alarab M, Regan C, O'Connell MP, Keane DP, O'Herlihy C, Foley ME. Singleton vaginal breech delivery at term: still a safe option. Obstet Gynecol 2004;103(03):407-412

8 ACOG. Mode of term singleton breech delivery. ACOG committee opinion. Available from: https://www.acog.org/Clinical-Guidance-and-Publications/Committee-Opinions/Committee-onObstetric-Practice/Mode-of-Term-Singleton-Breech-Delivery. Accessed February 22, 2019

9 ACOG Committee on Practice Bulletins-Obstetrics. Practice bulletin no. 161: external cephalic version. Obstet Gynecol 2016;127 (02):e54-e61

10 ACOG. The limits of conscientious refusal in reproductive medicine. ACOG committee opinion No. 385. Obstet Gynecol 2007; (110):e1203-e1208

11 Azria E, Le Meaux JP, Khoshnood B, Alexander S, Subtil D, Goffinet F; PREMODA Study Group. Factors associated with adverse perinatal outcomes for term breech fetuses with planned vaginal delivery. Am J Obstet Gynecol 2012;207(04):285.e1-285.e9

12 Zhang J, Troendle JF, Yancey MK. Reassessing the labor curve in nulliparous women. Am J Obstet Gynecol 2002;187(04):824-828

13 Zhang J, Troendle J, Reddy UM, et al; Consortium on Safe Labor. Contemporary cesarean delivery practice in the United States. Am J Obstet Gynecol 2010;203(04):326.e1-326.e10

14 Committee on Practice Bulletins-Obstetrics; Society for Maternal-Fetal Medicine. Practice bulletin no. 169. Multifetal gestations: twin, triplet, and higher-order multifetal pregnancies. Obstet Gynecol 2016;128(04):e131-e146

15 ACOG Committee on Ethics. Committee opinion no. 664: refusal of medically recommended treatment during pregnancy. Obstet Gynecol 2016;127(06):e175-e182

16 Arizona Department of State. Chapter 16. Department of health services-occupational licensing. 2017 September 2018; Available from: https://apps.azsos.gov/public_services/Title_09/9-16.pdf. Accessed February 22, 2019 
17 ACOG Committee on Obstetric Practice. Committee opinion no. 697: Planned home birth. Obstet Gynecol 2017;129(04):e117-e122

18 Wax JR, Lucas FL, Lamont M, Pinette MG, Cartin A, Blackstone J. Maternal and newborn outcomes in planned home birth vs planned hospital births: a metaanalysis. Am J Obstet Gynecol 2010;203(03):243.e1-243.e8

19 Woman, 36, who campaigned for home births dies having baby daughter at home. 2012; Available from: https://www.dailymail. co.uk/health/article-2094348/Caroline-Lovell-Home-birth-advocate-dies-delivering-baby-daughter-home.html. Accessed February 22,2019
20 ACOG committee opinion no. 559: Cesarean delivery on maternal request. Obstet Gynecol 2013;121(04):904-907

21 ACOG Committee on Obstetric Practice. Committee opinion no. 529: placenta accreta. Obstet Gynecol 2012;120(01): 207-211

22 Deneux-Tharaux C, Carmona E, Bouvier-Colle MH, Bréart G. Postpartum maternal mortality and cesarean delivery. Obstet Gynecol 2006;108(3, Pt 1):541-548

23 ACOG committee opinion no. 390, December 2007. Ethical decision making in obstetrics and gynecology. Obstet Gynecol 2007; 110(06):1479-1487 